\title{
El le invariable en el español escrito de Chile*
}

\author{
Guillermo Soto** \\ Scott Sadowsky*** \\ Ricardo Martínez $z^{* * * *}$
}

\begin{abstract}
Resumen
La falta de concordancia entre el clítico dativo le y el objeto indirecto (OI) plural, atestiguada tempranamente en español, sigue vigente. Las hipótesis que buscan explicarla apelan a la fonología, la economía morfosintáctica y la pragmática. Con el fin de comparar las realizaciones de le invariable y les concordante con OI en el español escrito de Chile, se analiza un corpus de 463 millones de palabras, considerando el orden de elementos en la oración y la distribución del fenómeno en variedades textuales. El estudio muestra que la frecuencia de le invariable depende tanto de la posición del OI como de propiedades de los contextos discursivos en que aparece. Se concluye que, de las tres hipótesis propuestas, solo la pragmática es compatible con los resultados.
\end{abstract}

Palabras clave: le invariable, le por les, concordancia, clítico dativo, objeto indirecto plural.

\section{Non-concordant le in written Chilean Spanish}

\begin{abstract}
The non-concordant use of the Spanish dative clitic le with a plural indirect object (IO) appeared early in Spanish and continues to occur to the present day. Phonetic, morphosyntactic and pragmatic explanations have been proposed to account for this phenomenon. The present paper analyzes a corpus of 463 million words of written Chilean Spanish for the purpose of studying the invariable (non-concordant) $l e$ and the concordant les used with plural IOs. Both their order of appearance in sentences and the phenomenon's distribution in different text types were analyzed. The results show that the frequency of the non-concordant $l e$ depends on both its position relative to the $\mathrm{IO}$ and certain properties of the discourse contexts in which it occurs. We conclude that of the three hypotheses, only the pragmatic one is compatible with the results of the study.
\end{abstract}

Key words: non-concordant $l e$, le for les, number agreement, dative clitic, plural indirect object.

Recibido: 01-03-2013

Aceptado: 18-07-2013

* Esta investigación fue apoyada por la Dirección de Investigación de la Facultad de Filosofía y Humanidades, Universidad de Chile. Una versión muy preliminar fue presentada en el Tercer Encuentro Nacional de Estudios del Discurso (Universidad Austral, Valdivia, Chile, 28 de septiembre a 1 de octubre de 2004).

** Chileno, Doctor en Filosofía y Letras, Universidad de Chile, Santiago, Chile, gsoto@uchile.cl

*** Estadounidense, Doctor en Lingüística, Universidad de La Frontera, Temuco, Chile, ssadowsky@ gmail.com

**** Chileno, Magíster en Estudios Cognitivos, Universidad de Chile y Universidad Diego Portales, Santiago, Chile, ricardomartinezg@gmail.com 
Por otra parte, sería también de interés conocer el comportamiento, cuando escribe, del hablante que oralmente emplea le por les.

Lidia Contreras

\section{Introducción}

El presente trabajo explora cuantitativamente, en un corpus escrito del español de Chile, la distribución relativa de oraciones semejantes a 1-4:

(1) Les dije a ellos.

(2) Le dije a ellos.

(3) A ellos les dije.

(4) A ellos le dije.

Como se advierte en los ejemplos, mientras en las oraciones (1) y (3) el dativo concuerda en número con el objeto indirecto (OI) expreso, en (2) y (4) no lo hace. Se trata, éste, de un fenómeno característico del español, advertido ya por Cuervo (1939), quien destacó al respecto que "entre los hechos que los gramáticos califican de errores, pocos hay que sean más geniales de nuestra lengua" (§335).

Contra la eventual creencia de que éste podría ser un fenómeno marginal, ausente en la tradición lingüística, baste mencionar ejemplos en Cervantes y Tirso, entre otros muchos autores, tanto peninsulares como americanos:

(5) Y si no me quejo del dolor es porque no es dado a los caballeros andantes quejarse de herida alguna, aunque se le salgan las tripas por ella ${ }^{1}$.

(El Quijote, §335)

1 Aunque, en Schevill y Bonilla (1928): “[s]in embargo, me inclino a creer que es un caso de concordancia con un sujeto mental singular, y no con lo escrito: no es dado a los caballeros andantes (es decir, a ningún caballero) quejarse de herida alguna, aunque se le salgan las tripas por ella" (460). Cfr. Sturgis (1927): "In every example cited by Sr. Casares or Sr. Rodríguez Marín, as well as those of Cuervo, we have a perfect possibility of a substitution for the le by a cada uno de or cualquiera de, etc." (252). Esta interpretación de corte distributivo ha sido desechada por la bibliografía (cfr. De Mello 1992). 
(6) Embarcarme quiero a España

Y darle a mis males fin.

(El burlador de Sevilla, I, IX)

Aun la Real Academia, en la Gramática de 1880, incluye un caso que, más tarde, corregirá (Fernández Ramírez 1978):

(7) Dale las lilas a las niñas.

Desde inicios del siglo pasado se han ofrecido variadas descripciones y explicaciones de este uso (v., entre otros, Pietsch 1911, Hanssen 1913, Sturgis 1927, Alonso y Henríquez Ureña 1984, Kany 1951, Fernández Ramírez 1951, 1978, Gili Gaya 1964, Montes 1965, Rojas 1977, Butt y Benjamin 1988, Fontanella de Weinberg 1992, De Mello 1992, López Morales 1992, Contreras 2005). No obstante, aún existen posiciones encontradas tanto con respecto a la extensión del fenómeno como, sobre todo, a su descripción y causa, que ha sido atribuida a la fonética, la economía morfosintáctica y la pragmática.

En el presente trabajo se exponen los resultados de una investigación que indagó en las incidencias sintácticas y genéricas que afectan su realización en el español escrito de Chile. Empleando la metodología de la lingüística del corpus, se analizó un corpus de 463.195 .781 palabras, constituido por $\sim 830.000$ textos escritos agrupados en diversos géneros, con el objeto de comparar las realizaciones de le invariable y de les concordante con el objeto indirecto. En este análisis, se tomaron en cuenta tanto el orden de los elementos en la oración como las variedades genéricas en que se da el fenómeno. Tras exponer los resultados distribuidos de acuerdo con las variables posición y género discursivo, éstos se interpretaron proponiendo que la frecuencia de le invariable es afectada por la posición del OI — como ya había sugerido Cuervo- y por el género discursivo. Se concluye que, a diferencia de la hipótesis pragmática, la hipótesis fonética y la de economía morfosintáctica, esta última en su versión más básica, no son compatibles con los resultados.

\section{Estudios previos}

En consonancia con la aseveración ya citada de Cuervo (1939), la RAE (Esbozo 1973: § 3.10.4b-e) señala, con respecto al uso de le por les, que "no 
es raro encontrar estas discordancias en periódicos, y aun en escritores de todas las épocas, de ambos lados del Atlántico", planteamiento reiterado en la Nueva gramática de la lengua española de 2009 (§35.2k). En cuanto a su extensión en España, Fernández Ramírez (1951) indica que se trata de un "fenómeno muy singular y esporádico", mientras que Seco (1986) afirma que es "bastante frecuente" y se da en hablantes y aun en escritores, como ya había expuesto Cuervo (1939). De Mello (1992), en el estudio más completo hasta ahora del fenómeno, observa, por su parte, que es más común en Sevilla que en Madrid.

En lo que respecta a América, Kany (1951), citando ejemplos de Argentina, Uruguay, Chile, Colombia, Venezuela, Nicaragua, Honduras, México y Cuba, asevera que, aunque no desusado en España, el fenómeno "seems to be much commoner everywhere in America" (107), juicio compartido, entre otros, por Oroz (1966), Alarcos (1994) y Camacho (1999)2. Más específicamente, Vaquero (1996) y López Morales (1992) destacan que es muy frecuente en el Caribe (v. los mismos juicios en Alba 2004, para República Dominicana, y Vaquero 2001, para Puerto Rico). Bentivoglio y Sedano (1992), por su parte, apuntan que es de "uso bastante común" en Venezuela (v. Sánchez Doreste 2005 para Caracas); mientras que Moreno de Alba (1992) informa que es "sumamente frecuente en el español de México", en consonancia con la amplia extensión indicada por Kany.

De especial interés resulta el estudio, ya referido, de De Mello (1992), donde se propone, a partir del análisis del corpus de la denominada "lengua culta" en grandes ciudades hispanoamericanas, una gradiente que va desde aquellas capitales en que el fenómeno está muy extendido hasta las que lo manifiestan de manera muy esporádica. En el primer extremo se encuentra el español hablado en dos ciudades caribeñas y una del norte de Sudamérica: San Juan de Puerto Rico, La Habana y Bogotá. Una frecuencia intermedia se observa en Santiago de Chile y Caracas. Menor aun es ésta en Buenos Aires y Ciudad de México. Finalmente,

2 No obstante su extensión actual, Sánchez Méndez (2003) sugiere que el fenómeno se habría generalizado en América recién hacia el siglo XIX, pues "las confusiones eran casi nulas todavía en el XVIII" (301). Montes (1965) sugiere cautela con respecto a la afirmación de que sería más común en América que en la península, considerando "la profusión de ejemplos que de España trae Cuervo" (623). De Kock (2002), en cambio, apoyándose en un corpus exiguo, afirma que sería una construcción propia del continente americano. 
tanto en Lima como en La Paz se da de manera esporádica. El estudio de ciudades españolas le permite al autor ubicar, como ya se anticipó, a Sevilla y Madrid en esta relación. La primera, en el nivel de Santiago de Chile y la segunda, en el de Lima.

Con respecto a la presencia del fenómeno en Chile, hacia 1912 Vicuña (cit. por Oroz) afirma que "el pronombre dativo oblicuo a la tercera persona se emplea siempre en singular, aun en lo escrito, cuando precede al complemento", y más tarde Lenz (1935), sin referirse directamente al esquema en que clítico y objeto indirecto aparecen simultáneamente, apunta que la /s / posnuclear "desaparece casi siempre, en el dativo plural, que es, también en la conversación de gente culta y en los diarios, con frecuencia igual al singular" (§ 156). Más recientemente, Oyanedel y Samaniego (1998-1999), en un estudio cuantitativo, concluyen que se trata de un fenómeno muy frecuente en lengua oral y escrita (véanse también Rabanales 1981, Sáez Godoy 1999, Cartagena 2002, Contreras 2005). Dado que, de acuerdo con sus datos, es más común en jóvenes $(70,9 \%)$ que en adultos (57\%), concluyen que estaría en proceso de generalización en la denominada "norma culta", proposición concordante con Penny $(2000)^{3}$. Contreras (1999) lo registra, también, en el español hablado por los mapuches.

Objeto de debate ha sido el papel que la posición relativa del OI desempeña en el fenómeno. Si bien ya Cuervo (1939) apuntaba que el fenómeno era más frecuente con OI pospuesto que antepuesto, postura reiterada en autores contemporáneos (Fernández Ramírez 1951, 1978; Fontanella de Weinberg 1992, De Mello 1992, RAE 2009), existen estudios que no consideran la variable (Oyanedel y Samaniego 1998-1999), que señalan expresamente que la posición del OI no incide (Quesada 1995), o que aseveran que el fenómeno se da solo con OI pospuesto (Vicuña 1912, Oroz 1966, Camacho 1999, Fernández Soriano 1999). RAE (2009) apunta a la eventual mayor presencia del fenómeno con OI que refieren a cosas.

Además del factor lingüístico, se han propuesto factores sociales para describir el uso. Así, la mayoría de los autores ha planteado que tendría

3 Esta proposición debe evaluarse con cautela por más de una razón. Primero, se trata, como se ha venido diciendo, de un fenómeno bastante antiguo y, en el caso de Chile, posiblemente ya extendido en el habla de los sectores socioeconómicos más elevados desde temprano, al menos en el siglo XX si se consideran los juicios de Vicuña y Lenz. Segundo, porque, como se sabe, conviene ser conservador en la proyección diacrónica de diferencias etarias. 
menor frecuencia entre personas de mayor nivel educacional. También se ha indicado que sería más frecuente en mujeres que en hombres (De Mello, 1992). Y mientras en su estudio del español de Chile, Oyanedel y Samaniego señalan que se da más en jóvenes que en adultos, De Mello concluye de sus datos que no habría diferencia relevante entre ambos grupos.

En lo que dice relación con el estatus normativo del fenómeno, no existe consenso entre los autores que consideran este alcance, aunque pareciera haber cierta tendencia, por parte de los estudios más actuales, a no censurarlo. Así, mientras Hanssen planteaba, en 1911, que era "propio de lengua vulgar", Cuervo lo trataba de "corruptela" y, más tarde, Alonso y Henríquez Ureña lo calificaban de "vicio idiomático" y Gili Gaya de construcción "disparatada", ya hacia 1970 Del Rosario señalaba que no es vicio idiomático. Interesante, en esta última línea, es la opinión de Butt y Benjamin (1988), quienes, junto con notar, como tantos otros, la alta frecuencia del empleo de le por "les redundante" en el mundo hispanohablante, destacan la existencia de casos en que el uso canónico es de dudosa aceptabilidad para el hablante culto (Él les da mucha importancia a las apariencias). En los últimos años, De Mello se limita a concluir que es más frecuente en habla no educada que educada; Oyanedel y Samaniego proponen, como ya se ha adelantado, que se trataría de un fenómeno en incorporación en la denominada "norma culta" chilena; Alba (2004) reconoce que "muchos hablantes, tanto en la República Dominicana como en otros países, están completamente seguros de que en ambos casos las formas empleadas son las correctas" ( $\mathrm{p}$. 322) y Penny (2000) concluye que el fenómeno compite fuertemente con la realización estándar. Con todo, aun en 1992 Moreno de Alba censura el uso como indebido y en 1999 Martínez acota que la construcción es "tachada de incorrecta" (§ 42.11). El Diccionario panhispánico de dudas (2005) lo considera desaconsejable desde el punto de vista normativo y RAE (2009), aunque reconoce su empleo en la lengua estándar "sobre todo en la oral" (§35.2k), aconseja mantener la concordancia "en registros formales".

Tres tipos de caracterizaciones se han adelantado con respecto al porqué del fenómeno: fonética, de economía morfosintáctica y pragmática. Probablemente no es de extrañar que Lenz haya caracterizado el uso en términos fonéticos, proponiendo la elisión de / s / final del dativo como una instancia más de la "debilidad" de la /s / posnuclear en el español 
de Chile. La idea de que en este caso la fonética guía la gramática, sin embargo, ha encontrado eco en otros autores como Keniston (1937), aunque con prevenciones, y los ya citados Oyanedel y Samaniego (19981999) y Contreras (2005). Frago (1999) es también de esa opinión, aunque con respecto a instancias recogidas de textos americanos del siglo XVI que no se ajustan del todo a los casos más comunes en la actualidad. ${ }^{4}$

La hipótesis fonetista, sin embargo, encuentra escollos difíciles de sobrepasar. Por un lado, el hecho de que la frecuencia del fenómeno varíe de acuerdo con la posición del OI, de ser efectivo, no puede explicarse sobre la base de argumentos fonéticos. Por otra parte, la existencia de usos en zonas en que la /s/ posnuclear no se aspira ni elide, como Ciudad de México (De Mello 1992), y los ejemplos hallados en Cervantes y otros autores, van en contra de lo que predice la hipótesis. Como tempranamente señaló Cuervo (1939), el fenómeno parece ser rasgo general del español y no exclusivo de zonas de debilitamiento de /s / posnuclear. Con todo, no es posible negar algún papel coadyuvante del factor fonético considerando que, como ya se ha indicado, los estudios sugieren que el uso tendería a ser más frecuente en zonas aspirantes. Lo que parece descartado, más bien, es que la causa del fenómeno sea de naturaleza fonética.

Diversos autores han descrito gramaticalmente el fenómeno, fundándolo, por lo general, bien en la analogía, bien en la economía comunicativa. Ya Casares (1918) propone una recategorización del clítico, que termina transformándolo en un mero índice anafórico o catafórico del OI; posición reiterada, en lo fundamental, por Gili Gaya (1964) y, posteriormente, por el Esbozo de la RAE (1973), que plantean que, en los contextos de OI posverbal, el clítico simplemente anticiparía o anunciaría vagamente el OI. Varios autores han destacado el papel que podría haber desempeñado en el origen del fenómeno la analogía con el dativo $s e(<g e)$, bajo el supuesto de que la neutralización de la oposición en le es paralela a la que se produjo con la neutralización de la misma oposición en se (se lo dije a él / se lo dije a ellos). Así, Keniston (1935), quien añade la analogía

4 Frago encuentra instancias tempranas del fenómeno en Hispanoamérica, para las que sugiere una causación fonética que, por el orden de los constituyentes en dos de las oraciones, no parece del todo inadecuada:

[A] Melchor Gonçález y a Alonso Gonçález le dezí que quando de acá no se enbiará nada. [E]n el sobrescrito le pone a las cartas un peso de porte

[A] buestro padre y madre le podés decir que por amor de Dios, que me perdonen y le da mis encomiendas. 
con el denominado se reflejo, y, más recientemente, Penny (2000), para quien el empleo invariable del le indirecto obedecería a una interferencia del alomorfo se. De acuerdo con De Mello (1992), Rini, en un trabajo al que no hemos tenido acceso, debilita esta hipótesis proponiendo que le por les sería, en realidad, un arcaísmo sintáctico ${ }^{5}$. El presente trabajo no explora la viabilidad de estas hipótesis.

Varios trabajos han destacado el papel que desempeñaría la economía comunicativa en la neutralización de la oposición le-les en contextos de $\mathrm{OI}$ léxico. Así, Montes (1965) explica el fenómeno por economía morfológica: "en la casi totalidad de los ejemplos aducidos [i. e., los catafóricos] el le es un simple signo redundante del dativo al cual no hace falta para nada el morfema de plural que está claramente expresado en otros términos" (624625). Por su parte, Quesada (1995), a partir de De Mello (1992), inscribe el fenómeno en el marco de la pluralización discontinua, proponiendo que gracias al principio de economía lingüística se generaría una neutralización del marcador de plural en el clítico. El fenómeno ocurriría, a juicio de este autor, independientemente de la posición relativa del clítico y el objeto indirecto. En la misma línea, Fernández Soriano (1999) propone una subespecificación del rasgo de número en clíticos dativos en construcciones reduplicativas. Pérez Silva (2000), asumiendo que el clítico marca concordancia de objeto indirecto, plantea que la presencia del objeto indirecto con rasgos $\left[+3^{\mathrm{a}},+\right.$ dativo, + plural, +léxico $]$ conllevaría la aplicación de una regla de desvinculación del nudo de número que impediría insertar el signo de plural, generándose, así, una oración con un clítico aparentemente singular.

Las caracterizaciones fundadas en la economía morfosintáctica predicen ya que la posición del OI es irrelevante, ya que OI antepuesto facilitaría el fenómeno toda vez que haría más patente la redundancia. Sin embargo, como se ha expuesto, la mayor parte de los estudios muestra que le por les es sensible a la posición del OI, e incluso que sería más frecuente con OI pospuesto (p. ej., Cuervo 1939, Sánchez Doreste 2005).

5 Contra parte de la bibliografía que, como se viene viendo en el presente trabajo, plantea más bien la novedad del fenómeno y aun su carácter de cambio en curso. Obsérvese, por ejemplo, a Lázaro Carreter (1992), para quien el le invariable es uno de los rasgos que caracterizan lo que denomina "visibles líneas de avance hacia el idioma del año 2000 ", tanto en su variedad hablada como escrita. Más cauto al respecto era, por cierto, el ya citado Cuervo. 
De Mello (1992) caracteriza el fenómeno desde una perspectiva pragmática, sosteniendo que el le invariable comunica una reducción del énfasis. En los casos con OI posverbal, distingue tres tipos de construcciones: sin clítico (dije a ellos), con clítico concordante (les dije a ellos) y con clítico invariable (le dije a ellos). Mientras la primera se asociaría con un énfasis elevado; la última lo haría con un énfasis bajo. La construcción con clítico concordante tendría un estatus intermedio. La menor frecuencia del fenómeno con OI antepuesto sería consistente con la propuesta, en la medida en que en estos casos estaríamos ante una rematización o focalización del OI, esto es, ante instancias de énfasis. No obstante, quedarían por explicar los casos en que le por les se da con OI antepuesto.

Weissenrieder (1995), desde una perspectiva próxima a la de De Mello sugiere que el fenómeno se correlacionaría con OIs no topicales; esto es, con sintagmas nominales de baja relevancia discursiva. En apoyo de su tesis, afirma que, de los ocho casos de le por les con OI plural en la novela El beso de la mujer araña, del argentino Manuel Puig, cinco correspondían a SNs referidos a inanimados y tres a referidos a animales o indefinidos, ninguno a un protagonista.

Aunque De Mello y Weissenrieder parecieran sostener aseveraciones incompatibles respecto del fenómeno (en un caso, índice de unidad fuera de foco; en el otro, índice de unidad no topical), ambos planteamientos podrían integrarse siguiendo la idea de Arnold (1999) en el sentido de que foco y tópico son nociones relacionadas en la medida en que las unidades en foco son justamente aquellas con alta probabilidad de transformarse en tópico discursivo y las unidades topicales corresponden a antiguos focos.

\section{Metodología}

Con el objeto de comparar las realizaciones de le invariable y de les concordante con OI en el español escrito de Chile, se analizó el Corpus Dinámico del Castellano de Chile (Sadowsky 2008), en su versión 2004, empleando metodologías de la lingüística de corpus. Se consideraron tanto el orden de los elementos en la oración (OI antepuesto vs. OI pospuesto) como las variedades genéricas en que se da el fenómeno.

El Codicach es un corpus de español escrito de carácter sintópico (los textos corresponden al español de Chile), sincrónico (98\% de los 
textos corresponden al período 1997-2003), profundo ( 450 millones de palabras y $\sim 500.000$ textos al momento de realizar el estudio; $\sim 800$ millones de palabras y 1,3 millones de textos en 2008), rotulado (incluye información sobre las fuentes textuales), electrónico (contiene textos digitales que permiten el acceso computacional al corpus) y modular (se pueden definir subcorpora ad hoc para cualquier finalidad).

En la medida en que se trata de un corpus de español escrito, el análisis permite aquilatar la hipótesis, por lo demás bastante debilitada en la actualidad, del carácter fonético del fenómeno. Por su parte, el contraste de la distribución de le por les de acuerdo con la posición del OI permite evaluar la incidencia sintáctica en el fenómeno y, más específicamente, la hipótesis de economía morfosintáctica, considerando una amplia base de datos. Por otro lado, el contraste de la distribución de le invariable por variedad de discurso posibilita evaluar la incidencia de parámetros como el control y la edición, y, a partir de ello, evaluar indirectamente el estatus normativo del fenómeno e inferir su grado de asentamiento. Finalmente, el tamaño del corpus permite contrastar resultados de investigaciones anteriores con los que arroja el análisis hecho sobre una amplia base de datos.

El análisis empleó los patrones que se observan en el Cuadro 1:

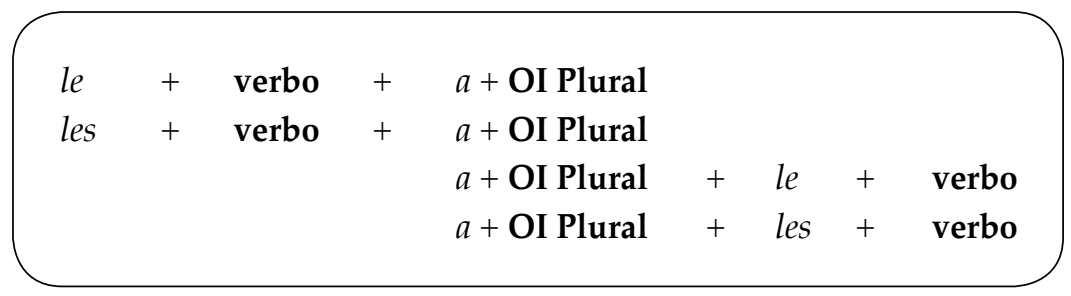

Cuadro 1: Patrones empleados en el análisis

Como se aprecia en el Cuadro 1, el análisis contempló las dos construcciones más fundamentales en que puede ocurrir el fenómeno: clítico + verbo + OI, por un lado, y $\mathrm{OI}+$ clítico + verbo, por otro. No se analizaron los casos en que intercede algún sintagma entre verbo y $\mathrm{OI}$ (le(s) dije de manera indirecta a los colegas; le(s) dije suavemente a los niños; le(s) dije, y lo quiero recalcar, a los dueños del edificio), ni aquellos casos en que clítico y OI aparecen en cláusulas distintas, como los siguientes, 
citados en Martínez (1999): Los niños hacen lo contrario de lo que le mandan los mayores; Si le dices que no vas, tus padres se van a preocupar.

En el Cuadro 2 se detallan las variedades textuales que se analizaron, contabilizando, además, el número de palabras correspondientes a cada una de ellas. Las variedades textuales se agrupan en dos categorías: textos sometidos a un proceso de edición profesional, y textos no sometidos a tal proceso:

\begin{tabular}{|c|c|c|c|}
\hline $\begin{array}{c}\text { Variedad textual } \\
\text { (sin edición profesional) }\end{array}$ & $\begin{array}{c}\mathrm{N}^{\circ} \\
\text { palabras }\end{array}$ & $\begin{array}{c}\text { Variedad textual } \\
\text { (con edición profesional) }\end{array}$ & $\begin{array}{c}\mathrm{N}^{\mathbf{0}} \\
\text { palabras }\end{array}$ \\
\hline $\begin{array}{l}\text { Mensajes en foros } \\
\text { generales de Internet }\end{array}$ & 7.428 .602 & $\begin{array}{r}\text { Cartas al director en } \\
\text { periódicos }\end{array}$ & 10.179.174 \\
\hline $\begin{array}{r}\text { Mensajes en foros de } \\
\text { periódicos }\end{array}$ & 14.994 .237 & $\begin{array}{r}\text { Textos de periódicos: } \\
\text { Santiago }\end{array}$ & 193.501.491 \\
\hline $\begin{array}{r}\text { Mensajes en grupos de } \\
\text { Usenet }\end{array}$ & 5.513 .269 & $\begin{array}{r}\text { Textos de periódicos: } \\
\text { norte de Chile }\end{array}$ & 46.635 .447 \\
\hline \multirow[t]{9}{*}{$\begin{array}{r}\text { Subtotal: Textos sin } \\
\text { edición }\end{array}$} & 27.936.108 & $\begin{array}{l}\text { Textos de periódicos: } \\
\text { centro de Chile }\end{array}$ & 37.802 .783 \\
\hline & & $\begin{array}{r}\text { Textos de periódicos: } \\
\text { sur de Chile }\end{array}$ & 54.442 .746 \\
\hline & & $\begin{array}{r}\text { Artículos en revistas de } \\
\text { computación }\end{array}$ & 5.315 .142 \\
\hline & & $\begin{array}{r}\text { Artículos en revistas } \\
\text { infantiles }\end{array}$ & 3.702 .932 \\
\hline & & $\begin{array}{r}\text { Artículos en revistas de } \\
\text { interés general }\end{array}$ & 17.550 .507 \\
\hline & & Textos académicos & 8.357 .037 \\
\hline & & Obras literarias & 946.530 \\
\hline & & Textos jurídicos & 18.710 .602 \\
\hline & & $\begin{array}{r}\text { Subtotal: Textos con } \\
\text { edición }\end{array}$ & 435.259 .673 \\
\hline
\end{tabular}

Cuadro 2: Variedades textuales analizadas

Como se observa en el Cuadro 2, se seleccionaron variedades sujetas canónicamente a cuidadosos procesos de edición (textos de periódicos 
y revistas, textos académicos, obras literarias y textos jurídicos) y variedades en que estos procesos típicamente no se dan (mensajes en foros de Internet y mensajes en grupos de Usenet). Todos los textos son de fuentes chilenas y comprenden el período 1997-2003.

Los mensajes en foros generales de Internet comprenden mensajes enviados a foros electrónicos chilenos de participación pública y corte más bien juvenil. Por su parte, los mensajes en grupos de Usenet corresponden a mensajes registrados en 32 de los llamados "grupos de noticias" de la jerarquía chile.* de Usenet, un sistema de comunicación en Internet que existe paralelamente a la Web (nótese que, pese a su nombre, los grupos de noticias de Usenet no se centran en lo noticioso; sus temáticas van desde música rock y literatura hasta deportes y sistemas operativos). A su vez, los mensajes en foros de diarios corresponden a aportes a foros electrónicos pertenecientes a medios de comunicación chilenos, en los que los temas se centran en la actualidad noticiosa. Con respecto a las cartas al director, estas son cartas enviadas por hablantes comunes a la mayoría de los diarios de Chile (ocho de Santiago, nueve del norte del país, cuatro del centro y siete del sur). Por otro lado, los textos de periódicos incluyen artículos periodísticos y columnas de opinión de los diarios impresos más importantes del país, además de varios medios de prensa exclusivamente electrónicos (en su conjunto, once de Santiago, seis del norte, tres del centro, siete del sur). A su vez, los artículos de revistas de computación han sido publicados en varios medios impresos y electrónicos que se dedican a la informática y tecnologías relacionadas (un total de cuatro); mientras que los artículos de revistas infantiles corresponden a textos de tres revistas destinadas a lectores menores de edad, y los de revistas de interés general a los contenidos de once revistas del ámbito noticioso y cultural. Por otra parte, los textos académicos consisten en artículos académicos y científicos escritos por profesionales de las ciencias aplicadas, naturales, sociales y humanidades. En cuanto a las obras literarias, estas contemplan creaciones tanto líricas (poemas) como narrativas (cuentos y novelas) de veinticuatro autores distintos. Finalmente, los textos jurídicos comprenden leyes y documentos extraídos de bases de datos de jurisprudencia administrativa y judicial. 


\section{Resultados}

Los resultados expuestos en el Gráfico 1 muestran que el uso de le por les es mayoritario con OI pospuesto $(52,3 \%)$, mientras que con OI antepuesto decae notablemente (11,3\%). Estos datos confirman el papel que desempeña la posición del OI en la frecuencia del fenómeno en estudio, corroborando el planteamiento de Cuervo (1939), Ramírez (1951, 1978), Fontanella de Weinberg (1992), De Mello (1992) y RAE (2009), que, por lo demás, es el dominante en la bibliografía. Consecuentemente, van en contra tanto de Quesada (1995), quien plantea la no incidencia de la posición del OI, como de Vicuña (1912), Oroz (1966), Camacho (1999) y Fernández Soriano (1999), quienes proponen que solo se da con OI pospuesto

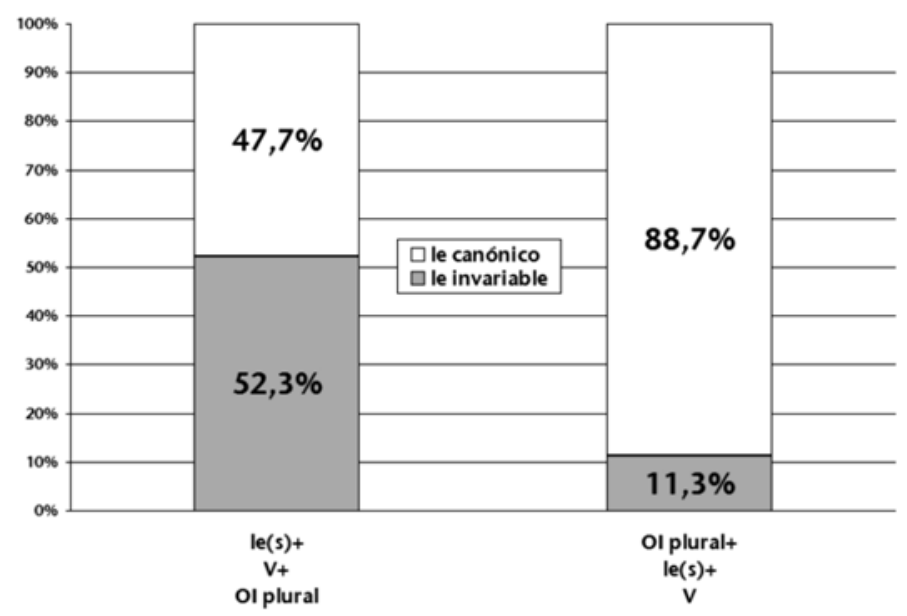

Gráfico 1: Incidencia de la posición del OI en le por les en la totalidad del corpus (463 millones de palabras).

Como se observa en el Gráfico 2, que da cuenta de los textos que no fueron sometidos a un proceso de edición profesional, la variedad textual no parece incidir mayormente en el fenómeno. Sin embargo, debe notarse que se dan sólo ocho casos de le por les con OI antepuesto en los foros generales de Internet, y sólo cuatro en los grupos de Usenet. 


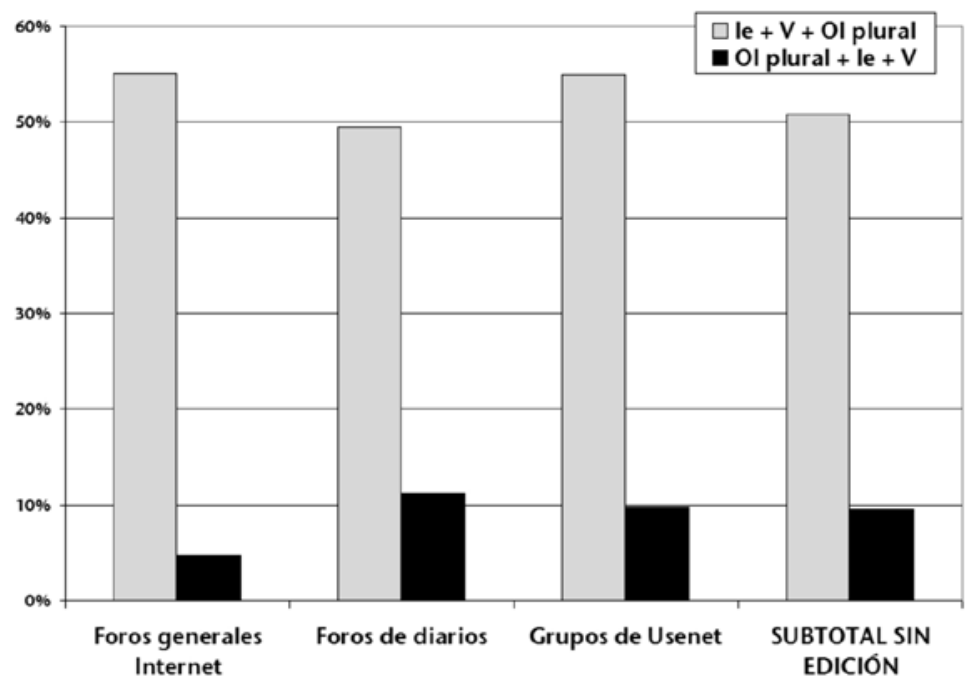

Gráfico 2: Incidencia de la variedad de discurso y la posición del OI en le por les: Textos no sometidos a un proceso de edición profesional (27,9 millones de palabras).

El caso de los textos sometidos a un proceso de edición personal se grafica en el Gráfico 3:

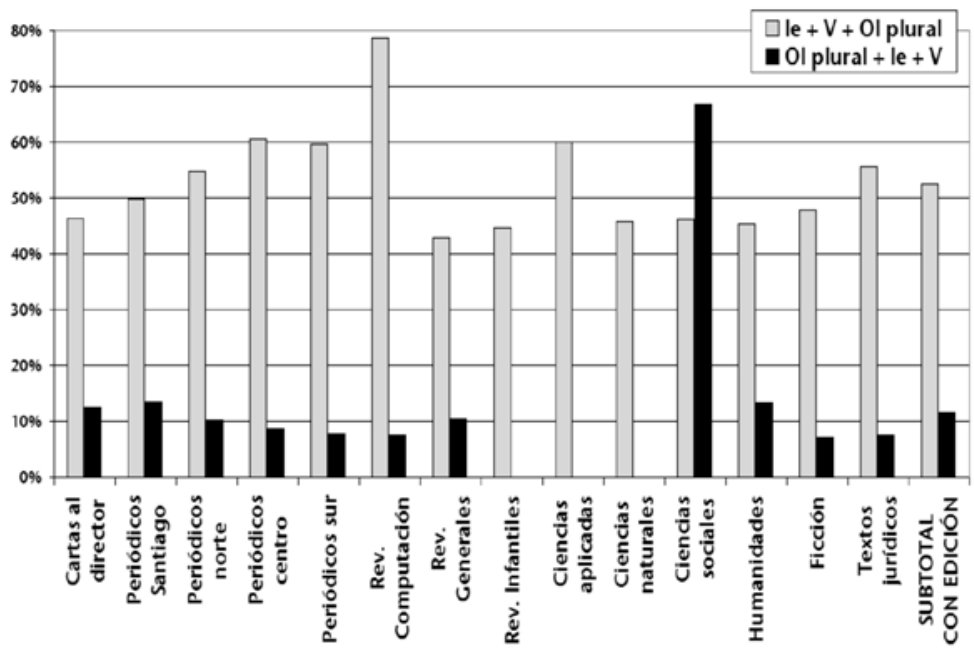

Gráfico 3: Incidencia de la variedad de discurso y la posición del OI en le por les: 


\section{Conclusiones}

Los resultados muestran que, en el español de Chile, el le invariable con OI pospuesto se encuentra bastante asentado en la lengua escrita en general (52\%), mientras que el le invariable con OI antepuesto lo está mucho menos (11\%). Aunque la proporción de le por les con OI pospuesto es inferior, en este estudio, a la que encuentran Oyanedel y Samaniego (1998-1999) en un corpus de lengua oral y escrita (57\% en adultos y 70,9\% en jóvenes), la presente investigación sugiere, en todo caso, que la posición del OI es el factor más importante de entre los observados.

Los resultados también sugieren que ciertas explicaciones que se han dado de le por les no serían adecuadas. En primer lugar, las explicaciones fonéticas, además de no explicar por qué el fenómeno se manifiesta en lengua escrita, predicen que la posición del OI sería irrelevante, lo que no concuerda con nuestros datos. Por otra parte, las explicaciones que apelan a la economía morfosintáctica parecieran predecir ya que la posición del OI es irrelevante ya que el OI antepuesto debería reforzar el fenómeno, hipótesis que no son confirmadas por los resultados. Ciertamente, puede plantearse, sustentando una versión más compleja de la hipótesis de economía morfosintáctica, que, en los casos de OI antepuesto, el OI se encuentra fuera de la cláusula nuclear, en una posición periférica que lleva a marcar como plural el dativo. Con todo, esta línea argumental demanda una explicación funcional de la extraposición del OI léxico, por lo que, a lo sumo, como indica De Mello (1992), en este caso la economía morfosintáctica puede constituir un factor coadyuvante en el fenómeno.

En contraposición con los planteamientos anteriores, hipótesis pragmáticas como la de Weissenrieder (1995), y, sobre todo, la de De Mello (1992) predicen correctamente que el fenómeno se refuerza en casos de OI pospuesto, aunque apelando a categorías funcionales distintas (tópico vs. foco). No explican, sin embargo, por qué se da un $11 \%$ de casos con OI antepuesto, toda vez que, en bruto, estas explicaciones - y en especial la de De Mello, que aborda específicamente el problema- predecirían un $0 \%$ de casos en dicho esquema. Parece necesario un análisis más fino que pueda, por una parte, discernir las condiciones de OI antepuesto con le invariable y concordante y, junto con ello, establecer el papel informativo o discursivo que desempeña el OI pospuesto en los casos de le por les. En principio, esperaríamos, ceteris paribus, que los OI léxicos en posición final tuviesen un estatus remático, tanto por su posición, típicamente 
asociada a un mayor dinamismo comunicativo, cuanto por el hecho de ser léxicos (cfr. Bolinger 1952, 1954-55, Contreras 1978, Gutiérrez Ordóñez 1997). El problema, por supuesto, es que, precisamente, esas condiciones se asocian con la información en foco (como contraria a la presupuesta). Hay, en consecuencia, que colegir que, en su planteamiento, De Mello distingue entre la posición no marcada de foco (en que sería esperable le por les) y la de foco marcado (en que se esperaría ausencia del clítico), situación, esta última, que denomina "de énfasis" (427).

En todo caso, las hipótesis de base pragmática no desechan, de suyo, la necesidad de una descripción del componente gramatical, toda vez que es justamente en ese ámbito donde se manifiestan las marcas que permiten reconocer la función del caso. Más aun, en la medida en que las caracterizaciones pragmáticas propuestas no parecen trasparentes, una interpretación de este tipo —aun suponiendo que se adecue a una observación más fina de los datos- resulta menos atractiva que una fundada en una concepción gramatical simbólica en que el valor pragmático/discursivo se asocie convencionalmente a cierto esquema morfosintáctico (una "gramática de construcciones", en el sentido de Croft y Cruse 2004. Cfr. también Langacker 2001, Goldberg 2003). Desde esta perspectiva, podría hipotetizarse que, en las construcciones en estudio, el dativo sufre una recategorización en virtud de la cual, como asevera Fernández Soriano (1999), ya "no ejerce una función verdaderamente pronominal, sino que avanza la presencia de un dativo, cuyos rasgos no necesita especificar" (§ 19.5.3. Véase también Demonte 2001). Dicha recategorización, sin embargo, no se interpretaría en el sentido de un le expletivo - fruto, a su vez, de un proceso de economía morfosintáctica, como sugiere la última parte de la cita de Fernández Soriano-, sino que se propondría, más bien, que esta se vincula convencionalmente a una función pragmático / discursiva específica asociada al estatus informativo o discursivo de la construcción con le invariable y OI pospuesto.

En cuanto a los factores que podrían afectar la distribución de le invariable por variedad textual, aparentemente, en los casos de OI pospuesto, el fenómeno sería menos frecuente en medios con mayor edición (leyes, revistas de interés general, diarios). Esto sugiere que, normativamente, todavía habría cierta censura con respecto al fenómeno, en consonancia con la postura del Diccionario panhispánico de dudas. En los casos de OI antepuesto, sin embargo, no se pudo establecer incidencia del tipo de medio. Es interesante destacar que los esquemas con OI 
antepuesto y pospuesto no parecen correlacionarse en los distintos medios considerados.

En general, de lo hasta aquí expuesto podemos concluir que el fenómeno se encuentra bastante extendido en lengua escrita; es mucho más frecuente con OI pospuesto que con OI antepuesto; no puede explicarse por razones fonéticas; no parece explicarse por mera economía lingüística; y podría obedecer a razones pragmáticas, como plantean De Mello y Weissenrieder, aunque esto último no implica asumir una postura pragmática radical.

El análisis también muestra que el esquema con OI pospuesto parece sensible a la fuente del discurso, posiblemente por la variable edición, mientras que el esquema con OI antepuesto no parece sensible a la fuente del discurso. Para una adecuada caracterización del fenómeno, y considerando los hallazgos de la presente investigación, es necesario realizar un estudio cualitativo que decida el eventual papel informativo o discursivo del OI léxico en cada una de las construcciones. 


\section{Referencias}

Alarcos, Emilio (1994). Gramática de la lengua española. Madrid: Espasa.

Alba, Orlando (2004). Cómo hablamos los dominicanos: un enfoque sociolingüístico. Santo Domingo: Grupo León Jiménez.

Alonso, Amado y Pedro Henríquez Ureña (1984). Gramática castellana. Segundo curso. Buenos Aires: Losada.

Arnold, Jenniffer E. (1999). "Marking salience: the similarity of topic and focus". Manuscrito disponible en Internet. Página web:

<http: / / www.unc.edu / jarnold / papers / Arnold_1999_MarkingSalience.doc>. (Consultado 10 de mayo de 2012)

Bentivoglio, Paola y Mercedes Sedano (1992). "Morfosintaxis". El idioma español de la Venezuela actual. Caracas: Cuadernos Lagovén, 46-70.

Bolinger, Dwight (1952). "Linear modification”. PMLA 67. 1117-1144.

Bolinger, Dwight (1954-1955). "Meaningful word order in Spanish". Boletín de Filología, VIII, "Homenaje a Rodolfo Oroz". 45-56.

Butt, John y Carmen Benjamin (1988). A new reference grammar of Modern Spanish. Londres: Arnold.

Camacho, José (1999). “La coordinación”, en I. Bosque y V. Demonte (dirs.). Gramática descriptiva de la lengua española, Vol. 2. Las construcciones sintácticas fundamentales. Relaciones temporales, aspectuales y modales. Madrid: Espasa, 2635-2694.

Cartagena, Nelson (2002). Apuntes para la historia del español en Chile. Santiago de Chile: Cuadernos de la Academia Chilena de la Lengua.

Casares, Julio (1918). Crítica efímera (Divertimentos filológicos). Madrid: Saturnino Calleja. 
Contreras, Constantino (1999). “El castellano hablado por mapuches: Rasgos del nivel morfosintáctico". Estudios Filológicos 34. 83-98.

Contreras, Heles (1978). El orden de palabras en español. Madrid: Ediciones Cátedra.

Contreras, Lidia (2005). "Usos pronominales no canónicos en el español de Chile", Onomázein 11 (1). 111-129. (Versión corregida de la publicada en Estudios Filológicos y Lingüísticos. Homenaje a Angel Rosenblat en sus 70 años. Caracas, Instituto Pedagógico, 1974. 157-172).

Croft, William y Alan Cruse (2004). Cognitive Linguistics. CUP: Cambridge University Press.

Cuervo, Rufino José (1939). Apuntaciones críticas sobre el lenguaje bogotano. $7^{a}$ edición. Bogotá: El Gráfico.

De Kock, Josse (2002). "Corpus, frecuencia y gramaticalidad. Treinta construcciones contrincantes en tres corpus", Romaneske 3, Página web: <http: / / www.vlrom.be/ pdf/023dekock.pdf>. (Consultado 10 de marzo de 2012)

De Mello, George (1992). "Le for les in the spoken educated Spanish of eleven cities", Canadian Journal of Linguistics 37. 407-430.

Demonte, Violeta (2001) “El español estándar (ab)suelto. Algunos ejemplos del léxico y la gramática". Ponencia presentada en el Segundo Congreso Internacional de la Lengua Española, “El español en la Sociedad de la Información". Valladolid, 16-19 de octubre. Página web: <http:/ / cvc.cervantes.es/obref/congresos/ valladolid / ponencias / unidad_diversidad_del_ espanol/1_la_norma_hispanica/demonte_v.htm>. (Consultado 3 de septiembre de 2003).

Fernández Ramírez, Salvador (1951). Gramática española. Los sonidos, el nombre y el pronombre. Madrid: Revista de Occidente. 
Fernández Ramírez, Salvador (1978). Problemas y ejercicios de gramática. Volumen ordenado y completado por Bienvenido Palomo Olmos. Madrid: Arco/Libros.

Fernández Soriano, Olga (1999). “El pronombre personal. Formas y distribuciones. Pronombres átonos y tónicos", en I. Bosque y V. Demonte (dirs.). Gramática descriptiva de la lengua española. Vol. 1 Sintaxis de las clases de palabras. Madrid: Espasa, 1208-1273.

Fontanella de Weinberg, María Beatriz (1992). El español de América. Madrid: Mapfre.

Frago, Manuel (1999). Historia del español de América. Textos y contextos. Madrid: Gredos.

Gili Gaya, Samuel (1964). Curso superior de sintaxis española. $9^{a}$ edición. Barcelona: Vox.

Goldberg, Adele (2003) "Constructions: a new theoretical approach to language". Trends in Cognitive Science 7 (5). 219-224.

Gutiérrez Ordóñez, Salvador (1997). Temas, remas, focos, tópicos y comentarios. Madrid: Arco Libros.

Kany, Charles (1951). American Spanish Syntax. $2^{a}$ edición, Chicago: The University of Chicago Press.

Langacker, Ronald (2001). "Discourse in Cognitive Grammar". Cognitive Linguistics 12. 143-88.

Lázaro Carreter, Fernando (1992). “La lengua española ante el año 2000”. Discurso de investidura como doctor honoris causa por la Universidad Carlos III de Madrid. Página web: <http: / / www.uc3m.es / uc3m / gral / DHC / thumbnails/1992_dis_fernando_lazaro_carreter. html>. (Consultado 3 de septiembre de 2003).

Lenz, Rodolfo (1935). La oración y sus partes. Estudios de gramática general y castellana. Madrid: Publicaciones de la Revista de Filología Española.

López Morales, Humberto (1992). El español del Caribe. Madrid: Mapfre. 
Martínez, José Antonio (1999). "La concordancia”, en I. Bosque y V. Demonte (dirs.). Gramática descriptiva de la lengua española, Vol. 2. Las construcciones sintácticas fundamentales. Relaciones temporales, aspectuales y modales. Madrid: Espasa, 2695-2786.

Montes, José Joaquín (1965). "Le por les ¿un caso de economía morfológica?". Thesaurus. Boletín del Instituto Caro y Cuervo XX. 622-625.

Moreno de Alba, José G. (1992). Minucias del lenguaje. México: FCE.

Moure, José Luis (2001). “Unidad y variedad en el español de América (Morfosintaxis)". Boletín de la Academia Argentina de Letras 261-262, LXVI. 339-356.

Oroz, Rodolfo (1966). La lengua castellana en Chile. Santiago: Facultad de Filosofía y Educación, Universidad de Chile.

Oyanedel, Marcela y José Luis Samaniego (1998-1999). “Notas para un nuevo perfil lingüístico del español de Santiago de Chile". Boletín de Filología XXXVII. 899-913.

Penny, Ralph (2000). Variation and change in Spanish. Cambridge: Cambridge University Press.

Pérez Silva, Jorge (2000). “Distribución y estructura interna de los clíticos del español: análisis de cuatro fenómenos desde la Morfología Distribuida". Lexis XXIV (2). 259-281.

Picallo, M. Carme y Gemma Rigau (1999). “El posesivo y las relaciones posesivas", en I. Bosque y V. Demonte (dirs.). Gramática descriptiva de la lengua española. Vol. 1, Sintaxis básica de las clases de palabras. Madrid: Espasa, 973-1023.

Quesada, J. Diego (1995). "Case, agreement and topicality in Spanish". Orbis. Bulletin international de documentation linguistique XXXVIII. 60-79.

Rabanales, Ambrosio (1981). "Perfil lingüístico de Chile", en H. Geckeler, B. Schlieben-Lange, J. Trabant, H. Weydt (eds.). Logos Semantikos: Studia Linguistica in honorem Eugenio Coseriu 1921-1981, Berlín, Nueva York, Walter de Gruyter. Madrid: Gredos, 447-464. 
Real Academia Española (1973). Esbozo de una nueva gramática de la lengua española. Madrid: Espasa-Calpe.

Real Academia Española (2005). Diccionario panhispánico de dudas. Madrid: Santillana.

Real Academia Española (2009). Nueva gramática de la lengua española. Madrid: Espasa.

Rojas, Nelson (1977). “Desajustes pronominales en español”, en Alan M. Gordon y Evelyn Rugg (eds.). Actas del VI Congreso de la Asociación Internacional de Hispanistas. 641-644.

Sadowsky, Scott (2008). Corpus Dinámico del Castellano de Chile (Codicach), Concepción (Chile), Universidad de Concepción. Página web: <www2.udec. $\mathrm{cl} / \sim$ ssadowsky/codicach.html $>$. (Consultado 10 septiembre 2008).

Sáez Godoy, Leopoldo (1999). El español de Chile en las postrimerías del siglo XX. Santiago: Bachillerato en Ciencias y Humanidades, Universidad de Santiago de Chile.

Sánchez Doreste, Rosa Elvira (2005). “Empleo de clíticos pronominales de tercera persona en el habla de Caracas". Revista de Ciencias Humanas y Sociales 21 (47). 37-61.

Sánchez Méndez, Juan (2003). Historia de la lengua española en América. Valencia: Tirant lo Blanch.

Schevill, Rodolfo y Adolfo Bonilla (1928). Obras completas de Miguel de Cervantes Saavedra, Don Qvixote, tomo I. Madrid: Gráficas Reunidas.

Sturgis, Cony (1927). “Uso de le por les”. Hispania 27. 251-254.

Vaquero, María (1996). “Antillas", en Manuel Alvar (ed.). Manual de dialectología hispánica. El español de América. Barcelona: Ariel.

Vaquero, María (2001). “Norma, normas y lenguaje informativo. Datos de Puerto Rico". II Congreso Internacional de la Lengua Española "El español en la Sociedad de la Información". Valladolid, 16-19 de octubre. Página web: <http:/ / cvc.cervantes.es/obref/congresos / 
valladolid/ponencias/unidad_diversidad_del_ espanol/1_la_norma_hispanica/vaquero_m.htm $>$. (Consultado 4 de diciembre de 2010).

Weissenrieder, Maureen (1995). "Indirect object doubling: saying things twice in Spanish". Hispania 78 (1). 169-177. 


\begin{tabular}{|c|c|c|c|c|c|c|c|c|c|}
\hline & \multicolumn{9}{|c|}{ LE INVARIABLE } \\
\hline & \multicolumn{3}{|c|}{ le $+v+$ ol plural } & \multicolumn{3}{|c|}{ OI plural + le + V } & \multicolumn{3}{|c|}{ le \& ol plural } \\
\hline & Casos & 96 & $\begin{array}{c}x \mathrm{mn} \\
\text { pal. }\end{array}$ & Casos & \% & $\begin{array}{c}x \mathrm{mn} \\
\mathrm{pal} .\end{array}$ & Casos & 9 & \begin{tabular}{|c|}
$x \mathrm{mn}$ \\
pal.
\end{tabular} \\
\hline \multicolumn{10}{|c|}{ SIN EDICIÓN PROFESIONAL } \\
\hline Foros generales Internet & 273 & $55,0 \%$ & 36,7 & 8 & 4,796 & 1,1 & 281 & $42,19 \%$ & 37,8 \\
\hline Foros de diarios & 1.066 & 49,596 & 71,1 & 56 & 11,296 & 3,7 & 1.122 & 42,396 & 74,8 \\
\hline Grupos de Usenet & 116 & $55,0 \%$ & 21,0 & 4 & 9,896 & 0,7 & 120 & 47,696 & 21,8 \\
\hline SUBTOTAL SIN EDICIO N & 1.455 & $50,8 \%$ & 52,1 & 68 & $9,6 \%$ & 2,4 & 1.523 & $42,6 \%$ & 54,5 \\
\hline \multicolumn{10}{|c|}{ CON EDICIÓN PROFESIONAL } \\
\hline Cartas al director & 329 & $46,3 \%$ & 32,3 & 20 & $12,4 \%$ & 2,0 & 349 & $40,0 \%$ & 34,3 \\
\hline PERIÓDICOS & & & & & & & & & \\
\hline Periodicos Santiago & 4.904 & 49,996 & 25,3 & 308 & 13,696 & 1,6 & 5.212 & 43,196 & 26,9 \\
\hline Periodicos norte & 1.022 & $54,7 \%$ & 21,9 & 46 & $10,1 \%$ & 1,0 & 1.068 & 46,096 & 22,9 \\
\hline Periodicos centro & 1.012 & $60,6 \%$ & 26,8 & 34 & 8,796 & 0,9 & 1.046 & $50,8 \%$ & 27,7 \\
\hline Periodicos sur & 1.388 & $59,7 \%$ & 25,5 & 45 & 7,796 & 0,8 & 1.433 & 49,396 & 26,3 \\
\hline Perto dicos & 8.326 & $53,1 \%$ & 22,5 & 433 & $11,7 \%$ & 1,2 & 8.759 & $45,2 \%$ & 23,6 \\
\hline \multicolumn{10}{|l|}{ REVISTAS } \\
\hline Rev. Computacion & 220 & $78,6 \%$ & 41,4 & 2 & 7,496 & 0,4 & 222 & 72,396 & 41,8 \\
\hline Rev. Cenerales & 469 & 42,996 & 26,7 & 32 & 10,396 & 1,8 & so 1 & 35,796 & 28,5 \\
\hline Rev. Infantiles & 33 & 44,696 & 8,9 & 0 & $0,0 \%$ & 0,0 & 33 & 37,996 & 8,9 \\
\hline Revistas & 722 & $49,9 \%$ & 27,2 & 34 & $9,7 \%$ & 1,3 & 756 & $42,1 \%$ & 28,5 \\
\hline \multicolumn{10}{|l|}{ T. ACADÉMICOS } \\
\hline Ciencias aplicadas & 3 & $60,0 \%$ & 6,0 & 0 & $0,0 \%$ & 0,0 & 3 & $60,0 \%$ & 6,0 \\
\hline Ciencias naturales & 11 & 45,89 & 2,7 & 0 & $0,0 \%$ & 0,0 & 11 & 44,096 & 2,7 \\
\hline Ciencias s $\propto$ ciales & 12 & 46,296 & 10,8 & 2 & 66,796 & 1,8 & 14 & 48,396 & 12,6 \\
\hline Humanidades & 20 & 45,596 & 7,3 & 2 & 13,396 & 0,7 & 22 & 37,396 & 8,1 \\
\hline Textos aca démlcos & 46 & $46,5 \%$ & 5,5 & 4 & $21,1 \%$ & 0,5 & so & $42,4 \%$ & 6,0 \\
\hline Ficcion & 22 & $47,8 \%$ & 23,2 & 1 & $7,1 \%$ & 1,1 & 23 & $38,3 \%$ & 24,3 \\
\hline Textos Juridicos & 133 & $55,6 \%$ & 7,1 & 5 & $7,6 \%$ & $\mathbf{0 , 3}$ & 138 & $45,2 \%$ & 7,4 \\
\hline \multicolumn{10}{|l|}{ TOTALES } \\
\hline SUBTOTAL SIN EDICIÓN & 1.455 & $50,8 \%$ & 52,1 & 68 & 9,696 & 2,4 & 1.523 & 42,696 & 54,5 \\
\hline SUBTOTAL CON EDICION N & 9.578 & 52,596 & 22,0 & 497 & 11,596 & 1,1 & 10.075 & 44,796 & 23,1 \\
\hline TOTAL GLO & 11.033 & $52,3 \%$ & 23,8 & 565 & $11,3 \%$ & 1,2 & 11.598 & $44,4 \%$ & 25,0 \\
\hline
\end{tabular}




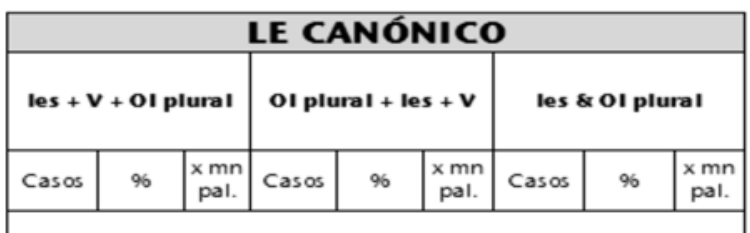

\begin{tabular}{|c|c|c|c|}
\hline \multicolumn{3}{|c|}{ TOTAL } & \multirow{3}{*}{$\begin{array}{c}\text { Palabras en } \\
\text { corpus }\end{array}$} \\
\hline $\begin{array}{l}l e(s)+ \\
\mathbf{v}+\mathbf{O} \mid \\
\text { plural }\end{array}$ & $\begin{array}{c}\text { ol } \\
\text { plura I+ } \\
\text { le }(s)+V\end{array}$ & $\begin{array}{c}\text { le (s) \& } \\
\text { OI } \\
\text { plura I }\end{array}$ & \\
\hline Casos & Casos & Casos & \\
\hline 496 & 171 & 667 & 7.428 .602 \\
\hline 2.155 & 499 & 2.654 & 14.994 .237 \\
\hline 211 & 41 & 252 & 5.513 .269 \\
\hline 2.862 & 711 & 3.573 & 27.936.108 \\
\hline
\end{tabular}

\begin{tabular}{|c|c|c|c|c|c|c|c|c|c|c|c|c|}
\hline 382 & $53,7 \%$ & 37,5 & 141 & $87,6 \%$ & 13,9 & 523 & $60,0 \%$ & 51,4 & 711 & 161 & 872 & 10.179 .174 \\
\hline 4.928 & 50,196 & 25,5 & 1.964 & 86,496 & 10,1 & 6.892 & 56,996 & 35,6 & 9.832 & 2.272 & 12. 104 & 193.501 .491 \\
\hline 845 & 45,396 & 18,1 & 408 & 89,996 & 8,7 & 1.253 & 54,096 & 26,9 & 1.867 & 454 & 2.321 & 46.635 .447 \\
\hline 657 & 39,496 & 17,4 & 356 & 91,39 & 9,4 & 1.013 & 49,296 & 26,8 & 1.669 & 390 & 2.059 & 37.802 .783 \\
\hline 938 & 40,396 & 17,2 & 538 & 92,396 & 9,9 & 1.476 & 50,796 & 27,1 & 2.326 & 583 & 2.909 & 54.442 .746 \\
\hline 7.368 & $46,9 \%$ & 19,9 & 3.266 & $88,3 \%$ & 8,8 & 10.634 & $54,8 \%$ & 28,7 & 15.694 & 3.699 & 19.393 & 370.497 .749 \\
\hline 60 & $21,4 \%$ & \begin{tabular}{l|l}
11,3 \\
\end{tabular} & 25 & $92,6 \%$ & 4,7 & 85 & 27,796 & 16,0 & 280 & 27 & 307 & 5.315 .142 \\
\hline 623 & 57,196 & 35,5 & 279 & 89,796 & 15,9 & 902 & 64,396 & 51,4 & 1.092 & 311 & 1.403 & 17.550 .507 \\
\hline 41 & 55,496 & 11,1 & 13 & $100,0 \%$ & 3,5 & 54 & 62,196 & 14,6 & 74 & 13 & 87 & 3.702 .932 \\
\hline 724 & $50,1 \%$ & 27,3 & 317 & $90,3 \%$ & 11,9 & 1.041 & $57,9 \%$ & 39,2 & 1.446 & 351 & 1.797 & 26.568 .581 \\
\hline 2 & 40,096 & 4,0 & 0 & 0,096 & 0,0 & 2 & 40,096 & 4,0 & 5 & 0 & 5 & 498.678 \\
\hline 13 & 54,296 & 3,2 & 1 & 100,096 & 0,2 & 14 & 56,096 & 3,5 & 24 & 1 & 25 & 4.011 .878 \\
\hline 14 & 53,896 & 12,6 & 1 & 33,396 & 0,9 & 15 & 51,796 & 13,5 & 26 & 3 & 29 & 1.115 .011 \\
\hline 24 & 54,596 & 8,8 & 13 & 86,796 & 4,8 & 37 & 62,796 & 13,5 & 44 & 15 & 59 & 2.731 .470 \\
\hline 53 & $53,5 \%$ & 6,3 & 15 & $78,9 \%$ & 1,8 & 68 & $57,6 \%$ & 8,1 & 99 & 19 & 118 & 8.357 .037 \\
\hline 24 & $52,2 \%$ & 25,4 & 13 & $92,9 \%$ & 13,7 & 37 & $61,7 \%$ & 39,1 & 46 & 14 & 60 & 946.530 \\
\hline 106 & $44,4 \%$ & 5,7 & 61 & $92,4 \%$ & 3,3 & 167 & $54,8 \%$ & 8,9 & 239 & 66 & 305 & 18.710 .602 \\
\hline 1.407 & 49,296 & 50,4 & 643 & 90,496 & 23,0 & 2.050 & 57,496 & 73,4 & 2.862 & 711 & 3.573 & 27.936 .108 \\
\hline 8.657 & $47,59 \%$ & 19,9 & 3.813 & $88,59 \%$ & 8,8 & 12.470 & 55,396 & 28,6 & 18.235 & 4.310 & 22.545 & 435.259 .673 \\
\hline 10.064 & $47,7 \%$ & 21,7 & 4.456 & $88,7 \%$ & 9,6 & 14.520 & $55,6 \%$ & 31,3 & 21.097 & 5.021 & 26.118 & 463.195 .781 \\
\hline
\end{tabular}


